JOURNAL DE PHYSIQUE IV

Colloque C7, supplément au Journal de Physique III, Vol. 1, décembre 1991

C $7-17$

\title{
MATERIALS FOR DIODE PUMPED SOLID STATE LASERS
}

\author{
L.L. CHASE, L.E. DAVIS, W.F. KRUPKE and S.A. PAYNE \\ University of California, Lawrence Livermore National Laboratory, P.O. Box 5508, L-490 Livermore, \\ CA 94550, USA
}

\begin{abstract}
The advantages of semiconductor diode lasers and laser arrays as pump sources for solid state lasers are reviewed. The properties desired for solid state laser media for various diode pumping applications are discussed, and the characteristics of several promising media are summarized.
\end{abstract}

\section{Introduction}

The availability of powerful and efficient semiconductor diode lasers and arrays is revolutionizing solid state laser technology. Diode lasers are efficient, compact, and reliable pump sources for solid state laser media. These advantages translate into greatly improved performance of diode pumped lasers over conventional flashlamp pumped devices. The purpose of this overview is to summarize the performance factors desired for solid state media for various laser applications and to discuss the properties of several promising media.

II. Diode Laser Characteristics [1]

Diode lasers have been developed that operate at wavelengths ranging from about $650 \mathrm{~nm}$ to beyond $30 \mu \mathrm{m}$. Of greatest importance for pumping solid state lasers are the diode lasers based on the III-V semiconductor compounds of $\mathrm{Al}, \mathrm{Ga}$, and In with $\mathrm{P}$ and As. Lasers based on these compounds are now available over a more or less continuous wavelength range from $650 \mathrm{~nm}$ to somewhat beyond $1.5 \mu \mathrm{m}$, although high-power and efficiency have been achieved so far only for $\mathrm{Al}_{x} \mathrm{Ga}_{1-x} \mathrm{As}$ and $\mathrm{In}_{x} \mathrm{Ga}_{1-x} \mathrm{As}$ compositions operating from about $800 \mathrm{~nm}$ to $1000 \mathrm{~nm}$. Electrical to optical efficiencies approaching $60 \%$ have been achieved, although the best high power array efficiencies are about $40 \%$. The output power for pulse lengths beyond about $10 \mu \mathrm{s}$ is limited by thermal effects, so diodes are peak power limited. This is an important characteristic, since the maximum total energy that can be obtained from a diode is determined by the duty cycle.

The output radiation of an ordinary diode is about one $\mathrm{nm}$ in bandwidth and has an anisotropic angular distribution of several tens of degrees perpendicular to the junction (diffraction limited by the $\sim 1 \mu \mathrm{m}$ thickness of the active region) and about 10 degrees in the plane of the junction (on the order of 10X diffraction limited). Linear arrays of diode lasers are available in $1-2 \mathrm{~cm}$ lengths, providing peak output powers of several hundred Watts. Two dimensional arrays of diode lasers can be fabricated by either stacking these linear arrays or by monolithic surface emitting arrays of diodes fabricated on a planar substrate. At the present time, stacks of linear arrays are the only available high power 2$\mathrm{D}$ arrays, although this may change as further development occurs with monolithic arrays. Several methods are being investigated to focus the rapidly diverging output beams of 
diode arrays into laser media. Focusing by cylindrical fibers, shaped fiber lenses, and linear microlens arrays has been used with the linear diode arrays and stacks. Two dimensional microoptics are being actively developed for 2-D arrays.

Thermal management is a key area of development for high power diode laser arrays. The diode threshold current increases and efficiency decreases with increasing temperature. Also, the output wavelength varies by a few tenths of a nanometer per degree $\mathrm{C}$, and this causes the wavelength to shift with average power output and to chirp during an output pulse. Two methods used for cooling of linear arrays and stacks are water cooled copper and diamond heatsinks [2] and water cooled silicon microchannel coolers [3]. With copper heatsinks, peak diode array power densities of over $1 \mathrm{~kW} / \mathrm{cm}^{2}$ are available for short pulses $(\sim 100 \mu \mathrm{s})$ and low duty cycles. On the other hand, peak and average powers of several hundred $W / \mathrm{cm}^{2}$ have been obtained with the microchannel cooling. At pulse lengths exceeding about $1 \mathrm{~ms}$, which are desirable for pumping media with long upper laser level lifetimes, these arrays have chirps of several nanometers or more. For efficient pumping in the presence of this chirp an adequate bandwidth is required for the pump transition of the laser medium.

\section{Advantages of Diode Pumping}

Diode lasers can be tuned, by varying either the composition of the active region or by changing the operating temperature, to match the wavelength of the pump transition. This property, and the highly directional nature of the focused output of diode lasers allows pumping efficiencies approaching $100 \%$, and parasitic effects like color center formation and absorption can be avoided. Since the peak optical density at the pump transition is much larger than the average value for flashlamp radiation, laser elements can be reduced in size, leading to more compact laser designs. If it is possible to pump the laser species into an energy level very near in energy to the upper laser level, very little excess energy is imparted to the medium in the pump transition. If the lower laser level is not too far above the ground state, the quantum defect can be very high. For $\mathrm{Nd}^{3+}$ pumped with $807 \mathrm{~nm}$ AlGaAs diodes, for example, the quantum defect is 0.76 , compared with an average quantum defect of about 0.6 for flashlamp pumping. For some other rare earths, the quantum defect can be larger than 0.9. This property means that there is much less heating of the medium, and thermal lensing and thermal fracture are mitigated. Finally, diode lasers are much more reliable than other pump sources. Lifetimes of over $10^{9}$ shots are common for AIGaAs and InGaAs diodes and arrays [3]. This overwhelming combination of advantages accounts for the rapid establishment of diode lasers as the pump source of choice for solid state lasers.

\section{Desired Properties of Diode Pumped Laser Media}

We distinguish between lasers operating in two generally different modes: 1)CW or free-running pulsed mode; 2) energy storage mode(Q-switched or pulse extracted). In the $\mathrm{CW}$ or free running mode, the threshold pump power is determined by the need to have the gain in the medium equal the cavity losses. The gain is proportional to the product of the emission cross section $\sigma_{e}$ and the population difference of the upper and lower laser levels. Since the population inversion is proportional to the upper laser level lifetime $\tau$ for four level laser media, the pump threshold power is proportional to $\sigma_{e} \tau$. The FüchtbauerLadenburg formula

$$
\sigma_{e} \tau_{r}=\frac{\lambda^{2}}{8 \pi n^{2} \Delta \bar{v}}
$$

relates $\sigma_{e}$ (suitably averaged over all polarizations) and the radiative lifetime $\tau_{r}$ to the wavelength $\lambda$, the refractive index $n$ and the effective linewidth of the laser transition, $\Delta v$. 
For a particular $\lambda$ it is clearly desirable to have as narrow a gain spectrum as possible (very few and narrow transitions). A very anisotropic cross section can also be an advantage since the laser output can be polarized along an axis of large cross section, whereas the cross section in Eq. (1) is averaged over all polarizations. Note that a price must be paid for tunability, since a large $\Delta v$ gives a lower $\sigma_{e} \tau$ product.

In the second mode of operation, energy storage, the lifetime of the upper laser level is of most importance, and the emission cross section must only be large enough to provide a comfortably low saturation fluence, $\Gamma_{S}=h c / \lambda \sigma_{e}$, to give efficient extraction at output fluences below the optical damage threshold of the medium and cavity optics. The importance of the lifetime is obvious; the total absorbed diode laser power required to store a given amount of energy is inversely proportional to $\tau$. If the value of $\sigma_{e}$ given by $\mathrm{Eq}$. (1) is substituted into the expression for the saturation fluence, it becomes

$$
\Gamma_{s} \cong \frac{h c}{\lambda \sigma_{\theta}} \sim \frac{\tau \Delta \bar{v}}{n^{2} \lambda^{3}}
$$

Note that long lifetimes and adequately low saturation fluences are much more likely at long wavelengths because of the $\lambda^{3}$ factor in Eq. (3). Finally, we note that at high energy storage densities, upconversion processes become more important than in $\mathrm{CW}$ operation, so a medium that performs well in CW operation may be poor for energy storage.

\section{Survey of Laser Media for Diode Laser Pumping}

A. Nd lasers

Table I shows some of the important spectroscopic properties of $\mathrm{Nd}^{3+}$ in a selection of fluoride and oxide crystals and a silicate glass. Note that $\sigma_{e} \tau$ varies by over a factor of ten among these crystals. Optical to optical efficiencies of over $40 \%$ have been achieved with longitudinally pumped TEMOO Nd:YAG lasers and $\mathrm{Nd}: \mathrm{YVO}_{4}$ [4]. Average powers of up to $70 \mathrm{~W}$ have been achieved in transversely pumped slabs. Other promising materials on

Table I: Spectroscopic properties of $\mathrm{Nd}^{3}+$ in some representative media

\begin{tabular}{|c|c|c|c|c|c|c|c|c|}
\hline Parameter & YAG & GSGG & YLF & $\mathrm{YVO}_{4}$ & Bel & LOS* & $\begin{array}{l}\text { ED-2 } \\
\text { Class }\end{array}$ & Yost \\
\hline $\begin{array}{l}\text { Pump wavelength, } \mathrm{nm} \\
\text { Pump cross section, } 10^{-20} \mathrm{~cm}^{2} \\
\text { Transitlon width, } \mathrm{nm} \\
{ }^{4} \mathrm{~F}_{32} \text { manlfold decay, } \mu \mathrm{sec}\end{array}$ & $\begin{array}{r}808 \\
6.2 \\
\sim 1 \\
260\end{array}$ & $\begin{array}{r}808 \\
6.4 \\
21 \\
280\end{array}$ & $\begin{array}{r}792 \\
1.9 \\
\sim 2 \\
570\end{array}$ & $\begin{array}{l}808 \\
6.9(\pi) \\
\sim 8 \\
100\end{array}$ & $\begin{array}{r}811 \\
11.6 \\
-6 \\
150\end{array}$ & $\begin{array}{r}817 \\
6.5 \\
-1.5 \\
100\end{array}$ & $\begin{array}{r}810 \\
2.2 \\
215 \\
360\end{array}$ & $\begin{array}{r}810 \\
10 \\
22 \\
240\end{array}$ \\
\hline Pump saturation flux, $\mathrm{kW} / \mathrm{cm}^{2}$ & 15 & 14 & 23 & 36 & 14 & 38 & 31 & 10 \\
\hline $\begin{array}{l}\text { Laser cross section, } \\
10^{-20} \mathrm{~cm}^{2} \text { (at } 1060 \mathrm{~nm} \text { ) }\end{array}$ & 28 & 13 & 21 & 87 & 8.5 & 100 & 2.8 & 10 \\
\hline$\sigma_{.} \tau$ (relative to Nd:YAG) & 1 & 0.50 & 1.6 & 1.2 & 0.17 & 1.37 & 0.14 & 0.32 \\
\hline
\end{tabular}

this list are $\mathrm{LiYF}_{4}(\mathrm{YLF}), \mathrm{YVO}_{4}$, and LOS. Crystals stoichiometric in $\mathrm{Nd}^{3+}$ are potential candidates for miniature diode pumped lasers. Kaminskii has tabulated the properties of many such crystals [6].

\section{B. Quasj-three-level Mid-IR Media Activated By Er. Tm. Ho and Yb}

The resonance transitions between the two lowest spin-orbit manifolds of $\mathrm{Er}, \mathrm{Tm}$, and Ho occur at wavelengths of $1.6 \mu \mathrm{m}, 1.9 \mu \mathrm{m}$, and $2 \mu \mathrm{m}$, respectively. In a number of crystals the upper levels have lifetimes exceeding $10 \mathrm{~ms}$, which is attractive for energy storage laser applications. Flashlamp pumped lasers employing these ions have been 
extensively investigated, but there has been little work on energy storage configurations. An important characteristic of these ions is that the lower laser level has a substantial thermal population at room temperature, and excited fractions, $\beta_{\min }$, of ten percent or more must be created in order to get net gain. Because of this property, there is also a theoretical maximum energy extraction efficiency, $\eta_{\max }$, which is given, for extraction pulses short compared with the lifetime, by [7]

$$
\eta_{\max }=1-\frac{\beta_{\min }}{\beta_{0}}=1-\left[\beta_{0}\left(1+\frac{Z_{g}}{Z_{\theta}} e^{\Delta E / k T}\right)\right]^{-1}
$$

where $Z_{g}$ and $Z_{e}$ are the partition functions of the upper and lower levels, $\beta_{0}$ is the initial fraction of ions pumped into the excited state, and $T$ is the temperature. $\Delta E=E_{f}-E_{i}$, where $E_{f}$ and $E_{i}$ are the energies of the upper and lower laser levels relative to the lowest crystal field levels of the upper and lower manifolds. In order to get high extraction efficiency. values of $\beta_{0}$ significantly larger than $\beta_{\min }$ are required. Extraction efficiencies in excess of $50 \%$ are predicted for realistic amplifier configurations of these media [8].

We measured the absorption and emission spectra of these ions in several oxide and fluoride crystals. The chosen crystals provide a broad range of crystal field environments for the rare earth ions, so they should comprise a good survey of the possible range of their spectroscopic properties. The spectra and a detailed discussion will be published elsewhere [9]. Table II summarizes the peak cross sections of emission components that have large values of $\Delta \mathrm{E}$. The best choice of output wavelength will depend on the cross section needed for the particular application, but in most cases, the choice made in Table II is judged to provide the optimum combination of $\sigma_{e}$ and $\Delta E$ for nanosecond to microsecond output pulses. It is very apparent that the cross sections of Er and Tm are

Table II: Values of $\sigma_{\text {eff }}$ and $\Delta \mathrm{E}$ for $\mathrm{Er}, \mathrm{Tm}$, and Ho

\begin{tabular}{|c|c|c|c|c|c|c|c|}
\hline \multirow{2}{*}{ Host } & \multicolumn{2}{|c|}{ Er } & \multicolumn{2}{|c|}{$\mathrm{Tm}$} & \multicolumn{2}{|c|}{$\mathrm{H}_{0}$} & \multirow{2}{*}{$\begin{array}{l}\text { Coord: } \\
\text { Number }\end{array}$} \\
\hline & $\sigma_{\text {et1 }}$ & $\Delta \mathrm{E}$ & $\sigma_{\text {ett }}$ & $\Delta \mathrm{E}$ & $\sigma_{\text {eff }}$ & $\Delta \mathbf{E}$ & \\
\hline $\mathrm{Y}_{3} \mathrm{Al}_{5} \mathrm{O}_{12}$ & 0.45 & 470 & 0.22 & 580 & 0.98 & 460 & 8 \\
\hline $\mathrm{LiYF}_{4}$ & 0.42 & 350 & 0.33 & 360 & 1.20 & 280 & 8 \\
\hline $\mathrm{BaY}_{2} \mathrm{~F}_{8}$ & 0.27 & 280 & 0.11 & 310 & $t .07$ & 310 & 8 \\
\hline $\mathrm{LaF}_{3}$ & 0.37 & 220 & 0.25 & 210 & 0.33 & 320 & -9 \\
\hline $\mathrm{KCaF}_{3}$ & 0.12 & 260 & 0.15 & 250 & 0.28 & 300 & 6 \\
\hline $\mathrm{YAIO}_{3}$ & 0.31 & 450 & 0.50 & 460 & 0.82 & 460 & 12 \\
\hline $\mathrm{La}_{2} \mathrm{Be}_{2} \mathrm{O}_{5}$ & 0.23 & 250 & 0.23 & 450 & 0.42 & 340 & -10 \\
\hline
\end{tabular}

substantially lower than those of Ho in the same crystals. This is attributable in part to the favorable distribution of oscillator strength for Ho, particularly in eightfold coordination, and also because the broadening of the crystal field levels by phonons tends to be smaller for Ho. Although our survey was done to search for new materials, it seems that the two well-known media, $\mathrm{Y}_{3} \mathrm{Al}_{5} \mathrm{O}_{12}$ (YAG) and $\mathrm{LiYF}_{4}$ (YLF), provide the best spectroscopic properties for all three ions.

Since these ions must be pumped to substantial inversion levels in order to get gain and high extraction efficiency, the saturation flux of the pump transitions $\phi_{\text {sat }}=h \mathrm{c} / \lambda \sigma_{\mathrm{p}} \tau$ is of importance. The data for the various ions in YAG are shown in Table lil. Note that the pump cross sections $\sigma_{p} \sim 10^{-20} \mathrm{~cm}^{2}$ are not large, but the long lifetimes of the upper levels lead to saturation fluxes of about $1 \mathrm{~kW} / \mathrm{cm}^{2}$, which can be obtained from high power 
Table 1II: Spectroscopic properties of the pump transition of rare earths in YAG

\begin{tabular}{|c|c|c|c|c|c|c|}
\hline Ion & Transition & $\begin{array}{c}\text { Pump } \\
\text { wavelength } \\
\text { (nm) }\end{array}$ & $\left(10^{-20} \mathrm{~cm}^{2}\right)$ & $\begin{array}{c}\tau_{\text {illuor }} \\
\text { (msec) }\end{array}$ & $\begin{array}{r}\Gamma_{\text {eat }}^{\text {peak }} \\
\left(\mathrm{J} / \mathrm{cm}^{2}\right)\end{array}$ & $\begin{array}{c}\phi_{\mathrm{sat}}^{\mathrm{mln}} \\
\left(\mathrm{kW} / \mathrm{cm}^{2}\right)\end{array}$ \\
\hline Er & ${ }^{4} I_{15 / 2} \rightarrow{ }^{4} I_{13 / 2}$ & 1470 & 1.4 & 6.9 & 10 & 1.4 \\
\hline $\mathrm{Tm}$ & ${ }^{3} \mathbf{H}_{6} \rightarrow{ }^{3} H_{4}$ & 1620 & 1.1 & 11 & 11 & 1.0 \\
\hline Ho & ${ }^{5} l_{8} \rightarrow{ }^{5} I_{7}$ & 1880 & 1.05 & 8 & 10 & 1.3 \\
\hline Yb & ${ }^{2} F_{7 / 2} \rightarrow{ }^{2} F_{5 / 2}$ & 941 & 0.75 & 1.3 & 28 & 22 \\
\hline Nd & ${ }^{4} l_{9 / 2} \rightarrow{ }^{4} F_{5 / 2}$ & 808 & 6.2 & 0.25 & 4 & 16 \\
\hline
\end{tabular}

diode arrays with little or no concentration by microoptics. The best pump transitions of these ions are at wavelengths $>1.5 \mu \mathrm{m}$, for which now high power arrays are not yet available. It seems likely that this situation will change, however, and the possibility of direct diode pumping of $\mathrm{Er}$, Ho and $\mathrm{Tm}$ should not be ignored. Cross relaxation can also be used to pump some of these ions. For example, the sensitization of $\mathrm{Ho}^{3+}$ by $\mathrm{Tm}^{3+}$, which can be pumped efficiently by AIGaAs diodes, has been the subject of many spectroscopic and laser performance studies. It should be noted that laser operation in a ground state depleted mode can provide some benefits in the form of very uniform pumping of a gain medium if the optical density at the pump wavelength can be made sufficiently large [7].

Another promising ion for diode laser pumping is $\mathrm{Yb}[7,10]$. This ion has only two manifolds in the $4 f$ configuration, so there are no complications anticipated from cross relaxation, upconversion, or excited state absorption. Some survey data for $\mathrm{Yb}$ in various oxide and fluoride hosts is shown in Table IV [11]. Once again YAG and YLF provide

Table IV: Spectroscopic properties of $\mathrm{Yb3}+$ in several fluoride and oxide crystals

\begin{tabular}{|c|c|c|c|c|}
\hline HOST & $\begin{array}{c}\text { Measured } \\
\tau_{\mathrm{em}}\end{array}$ & $\Delta E\left(\mathrm{~cm}^{-1}\right)$ & $\sigma_{e m}\left(10^{-20} \mathrm{~cm}^{2}\right)$ & $\boldsymbol{\beta}_{\min }$ \\
\hline $\mathrm{LaF}_{3}$ & 2.2 & 269 & 0.44 & 0.26 \\
\hline LiYF $_{4}$ & 2.2 & 483 & 0.81 & 0.11 \\
\hline $\mathrm{BaY}_{2}^{4} \mathrm{~F}_{\mathrm{a}}$ & 2.0 & $\sim 575$ & 0.67 & 0.07 \\
\hline $\mathrm{KCaF}_{3}$ & 2.7 & -650 & 0.22 & 0.05 \\
\hline $\mathrm{Fb}_{2} \mathrm{NaVF}_{6}$ & 10.8 & $\sim 449$ & 0.10 & 0.13 \\
\hline$K Y_{3} F_{10}$ & 2.1 & 325 & 0.47 & 0.21 \\
\hline $\mathrm{SrF}_{2}{ }^{* 10}$ & 9.7 & $\sim 589$ & 0.16 & 0.07 \\
\hline $\mathrm{BaF}_{2}^{*}$ & 8.2 & 539 & 0.14 & 0.09 \\
\hline $\mathrm{Y}_{3} \mathrm{Al}_{5} \mathrm{O}_{12}$ & 1.1 & 612 & 2.03 & 0.06 \\
\hline YAIO & 0.7 & 350 & 1.31 & 0.19 \\
\hline $\mathrm{Y}_{2} \mathrm{SiO}_{5}^{3}$ & 1.0 & $\sim 634$ & 0.33 & 0.06 \\
\hline
\end{tabular}

the best combination of lifetimes and emission cross sections for $\mathrm{Yb}$. Although $\mathrm{Yb}$ has generally lower values of $\beta_{\min }$, thus requiring lower fractional inversions than $\mathrm{Er}, \mathrm{Tm}$ and Ho, it has much shorter lifetimes in all the crystals. This leads to very high pump saturation fluxes, tens of $\mathrm{kW} / \mathrm{cm}^{2}$, as shown in Table III. Pumping of large laser elements will require some form of concentration of the output of diode arrays. One advantage of $\mathrm{Yb}$ is that it can be pumped with InGaAs strained-layer diodes, which have demonstrated very good performance recently. 


\section{Diode Pumped Tunable $\mathrm{Cr}^{3}+$ Lasers}

Recently, diode laser pumping of low-field $\mathrm{Cr}$ media has been investigated for alexandrite, LiCAF, and LiSAF [12]. Diode pumped $\mathrm{Cr}^{3+}$ lasers can provide tunability over the $700-1000 \mathrm{~nm}$ region, with the possibility of doubling into the blue. Since the principal red absorption band of $\mathrm{Cr}^{3}+$ peaks at about $650 \mathrm{~nm}$, visible AIGalnP diode lasers, which do not provide the high power and efficiency of AIGaAs diodes, have been used to pump $\mathrm{Cr}^{3+}$. Cr:LiSAF is particularly promising for this application, however, because it can be doped with very large $\mathrm{Cr}$ concentrations without detrimental effects due to cross relaxation or residual absorption by ion pairs. It has been shown that the pump absorption coefficient in the range of AlGaAs diode wavelengths can be made large enough to pump with those diodes, and much better performance is anticipated [13].

\section{Summary}

The field of diode pumped solid state lasers is evolving rapidly. So far the work has emphasized media and lasing transitions that were used previously with flashlamp pumping. Because of the different requirements and many advantages of diode laser pumping, it is likely that new approaches to pumping employing sensitizers, upconversion, and new diode laser pump wavelengths will considerably expand the available set of laser media.

Acknowledgment: This work was performed under the auspices of the U.S. Department of Energy by Lawrence Livermore National Laboratory under Contract No. W-7405-Eng-48.

\section{REFERENCES}

/1/ A thorough review is given by W. Striefer, D. R. Scifres, C. L. Harnagel, D. F. Welch, J. Berger, and M. Sakamoto, IEEE J. Quant. Elect. 24, 883 (1988).

12/ J. Haden, G. Harnagel, and J. Eudriz, OSA Proceedings on Advanced Solid State Lasers, Vol. 6. Hans P. Jenssen and George Dubé, eds. (Optical Society of America, Washington, D.C., 1991), p. 18.

13/ R. Beach, D. Mundinger, W. Benett, V. Sperry, B. Comaskey, and R. Solarz, Appl. Phys. Lett. 56, 2065 (1990).

$14 /$ R. A. Fields, T. S. Rose, M. E. Innocenzi, H. T. Yura, and C. L. Fincher, OSA Proceedings on Tunable Solid State Lasers, Vol. 5, Michael L. Shand and Hans P. Jenssen, eds. (Optical Society of America, Washington, D.C., 1989), p. 301.

15/ B. Comaskey, R. Beach, D. Mundinger, W. Benett, B. Freitas, D. Vanlue, G. Albrecht, and R. Solarz, Advanced Solid State Lasers Conference, Hilton Head, S.C., March 18-20, 1991.

16/ A. A. Kaminskii, Laser Crystals (Springer-Verlag, Berlin, 1981), p. 362.

II W. F. Krupke and L. L. Chase, Optical and Quantum Electronics 22, 51 (1990).

18/ L. L. Chase, Stephen A. Payne, L. K. Smith, W. L. Kway, and W. F. Krupke, OSA Proceedings on Advanced Solid State Lasers, Vol. 7, to be published.

19/ Stephen A. Payne, L. L. Chase, L. K. Smith, Wayne L. Kway, and William F. Krupke, submitted to IEEE J. Quant. Elect.

110/ P. Lacovara, C. A. Wang, H. K. Choi, R. L. Aggarwal, and T. Y. Fan, QSA Proceedinas on Advanced Solid State Lasers, Vol. 7, to be published.

111/ Laura Davls, Stephen A. Payne, L. L. Chase, L. K. Smith, Wayne L. Kway, and William F. Krupke, to be published.

112/ See, for example, R. Scheps, J. F. Myers, H. Serreze, A. Rosenberg, R. C. Morris, and $M$. Long, Opt. Lett. 16, 830 (1991), and references therein.

/13/ Stephen A. Payne, William F. Krupke, Larry K. Smith, Wayne L. Kway, Laura E. Davis, and John B. Tassano, to be published. 